\title{
Free subgroups of inverse limits of iterated wreath products of non-abelian finite simple groups in primitive actions
}

\author{
Felix Leinen and Orazio Puglisi
}

Communicated by Andrea Lucchini

\begin{abstract}
Let $\mathcal{W}=\left\{G_{i} \mid 1 \leq i \in \mathbb{N}\right\}$ be a set of non-abelian finite simple groups. Set $W_{1}=G_{1}$ and choose a faithful transitive primitive $W_{1}$-set $\Delta_{1}$. Assume that we have already constructed $W_{n-1}$ and chosen a transitive faithful primitive $W_{n-1}$-set $\Delta_{n-1}$. The group $W_{n}$ is then defined as $W_{n}=G_{n} \mathrm{wr}_{\Delta_{n-1}} W_{n-1}$. If $W$ is the inverse limit $W=$ $\lim \left(W_{n}, \rho_{n}\right)$ with respect to the natural projections $\rho_{n}: W_{n} \rightarrow W_{n-1}$, we prove that, for $\overleftarrow{\text { each }} k \geq 2$, the set of $k$-tuples of $W$ that freely generate a free subgroup of rank $k$ is comeagre in $W^{k}$ and its complement has Haar measure zero.
\end{abstract}

\section{Introduction}

In 1971, the following theorem was proved by D. B. A. Epstein [5]:

Let $G$ be a connected, finite-dimensional nonsolvable Lie group. Then, for each $k>0$ and for almost all $k$-tuples $\left(g_{1}, \ldots, g_{k}\right)$ of elements in $G$, the group generated by $g_{1}, \ldots, g_{k}$ is free of rank $k$.

Here, almost all $k$-tuples means that the set of $k$-tuples in $G^{k}$, which do not generate a free group of rank $k$, form a set of measure zero in $G^{k}$ with respect to the Haar measure on $G$.

Epstein's result is the starting point of a series of papers in which the phenomenon of the so-called ubiquity of free groups is considered. Roughly speaking, it has been noticed that, for many groups $G$, the set $G(k)$ of $k$-tuples $\left(g_{1}, \ldots, g_{k}\right) \in G^{k}$ such that $\left\langle g_{1}, \ldots, g_{k}\right\rangle$ is free of rank $k$, is very large. Typically, the largeness of $G(k)$ is expressed by the fact that, either the group $G$ carries a natural topology and $G(k)$ is co-meagre (i.e., its complement is a meagre set), or $G$ is a measure space and the complement of $G(k)$ is a set of measure zero. In such cases we say, that almost every $k$-tuple of elements in $G$ generates a free subgroup of rank $k$.

The next author who investigated the ubiquity of free subgroups was J. D. Dixon in [3]. He studied the case $G=\operatorname{Sym}(\mathbb{N})$. The pointwise stabilizers in $G$ of the finite subsets of $\mathbb{N}$ form a basis of open neighborhoods of the identity, and this 
gives rise to a natural topology on $G$. Dixon proved the strong result that, for every $k \geq 2$, the set

$$
\begin{aligned}
H(k)=\left\{\left(g_{1}, \ldots, g_{k}\right) \in G^{k} \mid\right. & \left\langle g_{1}, \ldots, g_{k}\right\rangle \text { is free of rank } k \\
& \text { and acts highly transitively on } \mathbb{N}\}
\end{aligned}
$$

is co-meagre in $G^{k}$.

In 2003 the question about abundance of free subgroups was taken up by P. M. Gartside and R. W. Knight [6] from a different point of view. For Polish groups the authors find several conditions which are equivalent to co-meagreness of $G(k)$. Amongst others they show that, if $G$ is non-abelian and contains a dense free subgroup, then $G(k)$ is always co-meagre.

An interesting consequence can be drawn using a result of H. D. Macpherson in [9]. He proved that the automorphism group of any $\boldsymbol{\aleph}_{0}$-categorical structure contains a dense free subgroup of countable rank. Since $\operatorname{Aut}(M)$ is a Polish group whenever $M$ is a countable first order structure, it turns out that, for every $\aleph_{0}$-categorical structure $M$, almost every $k$-tuple of elements of $\operatorname{Aut}(M)$ generates a free group of rank $k$. In particular, this holds for some well-known structures like the ordered set $\mathbb{Q}$ or the random graph.

The starting point of our investigation is a paper of M. Bhattacharjee [2], in which the question about abundance of free subgroups is addressed for a class of groups consisting of inverse limits of certain wreath products. Consider the set $\mathcal{W}=\left\{\left(G_{i}, \Delta_{i}\right) \mid 1 \leq i \in \mathbb{N}\right\}$, where the group $G_{i}$ acts faithfully and transitively on $\Delta_{i}$ for each $i$. For every $n \in \mathbb{N}$, let $\Sigma_{n}=\Delta_{n} \times \cdots \times \Delta_{1}$. Now $W_{1}=G_{1}$ acts naturally on $\Sigma_{1}$. Thus, assuming that we have already defined $W_{n-1}$ and noticed that it acts naturally on $\Sigma_{n-1}$, we let $W_{n}=G_{n} \operatorname{wr}_{\Sigma_{n-1}} W_{n-1}$. Clearly $W_{n}$ acts naturally on $\Sigma_{n}$. With $\rho_{n}: W_{n} \rightarrow W_{n-1}$ denoting the natural projection map, we can form the inverse limit $W=\lim \left(W_{n}, \rho_{n}\right)$. For each $n$ there is an epimorphism $\sigma_{n}: W \rightarrow W_{n}$, and the set $\left\{\operatorname{ker}\left(\sigma_{n} \overleftarrow{)} \mid n \geq 1\right\}\right.$ can be taken as a basis of open neighborhoods of 1 . Bhattacharjee proved that, with respect to this topology, $W(k)$ is co-meagre for each $k$. Moreover, if all the groups $G_{i}$ are finite, then $W$ is a profinite group, so that it carries a Haar measure. In this case the complement of $G(k)$ is a set of measure zero for all $k \geq 2$. Improvements have been later obtained by other authors, see [1] and [15], using different methods.

In the present paper we study groups which can be constructed in a similar fashion. Let $\mathcal{W}=\left\{G_{i} \mid 1 \leq i \in \mathbb{N}\right\}$ be a set of non-abelian finite simple groups $G_{i}$. Let $W_{1}=G_{1}$ and choose a faithful transitive primitive $W_{1}$-set $\Delta_{1}$. Assume that we have already constructed $W_{n-1}$ and chosen a transitive faithful primitive $W_{n-1}$-set $\Delta_{n-1}$. The group $W_{n}$ is then defined as $W_{n}=G_{n} \mathrm{wr}_{\Delta_{n-1}} W_{n-1}$. As above, we can form the inverse limit $W=\lim \left(W_{n}, \rho_{n}\right)$ with respect to the natural projections $\rho_{n}: W_{n} \rightarrow W_{n-1}$. 
Inverse limits of wreath products of finite simple groups have been studied in several paper. J.S. Wilson [16] produced examples of hereditarily just-infinite profinite groups by means of inverse limits of wreath products of finite simple groups. This construction has been generalized recently by M. Vannacci [14]. The groups constructed by these authors are, in fact, of a quite particular nature. A characterization of (hereditarily) just infinite profinite groups is given in [12], where C. Reid finds necessary and sufficient conditions for a profinite group to be just infinite or hereditarily just infinite, in terms of the inverse system of its finite images. On the other hand (hereditarily) just infinite profinite groups form a pretty large class and very few general properties are known. Therefore specific constructions like the ones cited above, are of interest.

Inverse limits of wreath products of simple groups have been considered also by M. Quick, who showed in [10] and [11] that such inverse limits (when at each stage the wreathing action is transitive and faithful) are positively finitely generated. He gives lower bounds for the probability that two randomly chosen elements generate such a group $W$. The problem of finding an explicit set of generators was considered subsequently by Vannacci [13].

We study the abundance of free subgroups of the groups $W$ and prove that, for each such group and each $k \geq 2$, the set $W(k)$ is co-meagre and its complement has measure zero with respect to the Haar measure (Theorem 3.1). Our methods rely upon the ones developed in [2], but the fact that the primitive sets $\Delta_{n}$ can be chosen freely, requires a different kind of approach.

\section{Non-triviality of words in iterated wreath products}

In this paper, we shall consider the following classes.

- $W_{1}$ shall be the class of all pairs $(G, \Gamma)$, where $G$ is a non-abelian finite simple group and $\Gamma$ denotes some primitive faithful $G$-set.

- For every $n>1$, the class $\mathcal{W}_{n}$ shall consist of all pairs $\left(S \operatorname{wr}_{\Delta} G, \Gamma\right)$ such that

(i) $S$ is a non-abelian finite simple group,

(ii) $(G, \Delta) \in \mathcal{W}_{n-1}$, and

(iii) $\Gamma$ is a faithful primitive $\left(S \mathrm{wr}_{\Delta} G\right)$-set.

Consider the free group $F_{k}$ of rank $k$ with free generators $x_{1}, x_{2}, \ldots, x_{k}$. We shall make use of the following notation. If $w=y_{1}^{\epsilon_{1}} y_{2}^{\epsilon_{2}} \ldots y_{n}^{\epsilon_{n}}$ is a reduced word of length $n$ with $y_{i} \in\left\{x_{j} \mid j=1, \ldots, k\right\}$ and $\epsilon_{i}= \pm 1$, we let

- $w_{0}=1$ and $w_{i}=y_{1}^{\epsilon_{1}} y_{2}^{\epsilon_{2}} \ldots y_{i}^{\epsilon_{i}}$ for $i=1, \ldots, n$,

- $\bar{w}_{i}=w_{i}\left(x_{1}, \ldots, x_{k}\right)^{-1}$ if $\epsilon_{i}=-1$ and $\bar{w}_{i}=w_{i-1}\left(x_{1}, \ldots, x_{k}\right)^{-1}$ if $\epsilon_{i}=1$. 
For $k$-tuples $g=\left(g_{1}, \ldots, g_{k}\right)$ and $h=\left(h_{1}, \ldots, h_{k}\right)$ from $G^{k}$ we shall denote the $k$-tuple $\left(g_{1} h_{1}, \ldots, g_{k} h_{k}\right)$ by $g h$. Moreover, for every pair of element $a, b$ of a group, we shall indicate by $a^{b}$ the element $b^{-1} a b$.

The proof of the following lemma is straightforward.

Lemma 2.1. Let $w=x_{i_{1}}^{\epsilon_{1}} x_{i_{2}}^{\epsilon_{2}} \ldots x_{i_{n}}^{\epsilon_{n}}$ be a reduced word of length $n$ in $F_{k}$ and let $G$ be any group with a normal subgroup $N$. Consider $\left(g_{1}, \ldots, g_{k}\right) \in G^{k}$ and $\left(b_{1}, \ldots, b_{k}\right) \in N^{k}$. Then

$$
w\left(b_{1} g_{1}, \ldots, b_{k} g_{k}\right)=a_{1}^{\epsilon_{1}} \ldots a_{n}^{\epsilon_{n}} w\left(g_{1}, \ldots, g_{k}\right),
$$

where $a_{j}=\left(b_{i_{j}}\right)^{\bar{w}_{j}\left(g_{1}, \ldots, g_{k}\right)}$ for $j=1, \ldots, n$.

Lemma 2.2. Let $(G, \Gamma)$ be an element of $\mathcal{W}_{n}$ for some $n \geq 2$, with $G=S \mathrm{wr}_{\Delta} H$, where $(H, \Delta) \in W_{n-1}$. Then one of the following holds:

(1) The action of $G$ on $\Gamma$ is the diagonal action.

(2) $\Gamma=\Sigma^{\Delta}$ for a suitable primitive $S$-set $\Sigma$, and the action of $G$ on $\Gamma$ is the product action.

(3) $G$ is isomorphic to a twisted wreath product $S \operatorname{wr}_{\Theta} X$ with $X \simeq H$, and the socle $S^{\Theta}$ of $G$ acts regularly on $\Gamma$.

Proof. The socle of $G$ is the base group $B=S^{\Delta}$, and it acts transitively on $\Gamma$. If the action of $B$ is regular, then $G$ is the semidirect product of $B$ and $G_{\gamma}$ for any $\gamma \in \Gamma$. Moreover, $X=G_{\gamma}$ acts on the components of $B$ in the same way as $H$. By [7], the group $G$ is isomorphic to a twisted wreath product $S \operatorname{wr}_{\Theta} X$ in this case.

We may thus assume that $B$ is not regular, so that $B_{\gamma} \neq 1$. Since $|\Delta|>1$, we can use [4, Theorem 4.6.A] to see that one of the following cases must hold: either $G$ is of diagonal type, or $G$ is a primitive subgroup of $U \mathrm{wr}_{\Lambda} \operatorname{Sym}(\Lambda)$ acting in product action and $U$ is itself primitive with non-regular socle. In order to complete the proof, it suffices to show that $U=S$ and $\Lambda=\Delta$ in the latter case.

But then, $\operatorname{soc}(U)^{\Lambda}$ is the socle of $G$, so that the components of $\operatorname{soc}(U)$ form a block under the action of $G$ on the components of $B$. This action is primitive, so that $\operatorname{soc}(U)$ must have just one component. Therefore $U$ is isomorphic to a subgroup of $\operatorname{Aut}(S)$ containing $S$, so that $U / S$ is solvable. The normal closure $U^{G}$ of $U$ contains $B$ and it is easy to see that $U^{G} / B$ is solvable. However, $H$ has no non-trivial solvable normal subgroups. This shows that $U^{G}=B$ and $U=S$.

Consider some $(G, \Gamma) \in \mathcal{W}_{n}$. In order to give a lower bound for the size of $\Gamma$, we define the function $\xi: \mathbb{N} \rightarrow \mathbb{N}$ recursively via

$$
\xi(0)=1 \quad \text { and } \quad \xi(n)=5^{\xi(n-1)} \text { for all } n \geq 1 .
$$


Lemma 2.3. Using the above notation,

$$
\xi(n) \leq|\Gamma| \text { and } \quad\left(\begin{array}{c}
n+1 \\
2
\end{array}\right)<3^{\sqrt{\xi(n-1)}-2} \text { for all } n \geq 3 .
$$

Proof. The first claim holds true for $n=1$, because the symmetric group of degree 4 is solvable. If $\left(S \operatorname{wr}_{\Delta} H, \Gamma\right) \in W_{n}$ and $\xi(n-1) \leq|\Delta|$ by induction, then Lemma 2.2 implies that $|\Gamma| \geq 5^{|\Delta|} \geq 5^{\xi(n-1)}=\xi(n)$.

The second inequality holds true for $n=3$. For $n \geq 4$, induction gives

$$
\begin{aligned}
\left(\begin{array}{c}
n+1 \\
2
\end{array}\right) & =\frac{1}{2}\left((n+1)^{2}+(n+1)\right)=\frac{1}{2}\left(\left(n^{2}+n\right)+2(n+1)\right) \\
& <\frac{3}{2}\left(n^{2}+n\right)=3\left(\begin{array}{l}
n \\
2
\end{array}\right)<3^{\sqrt{\xi(n-2)}-1}<3^{\sqrt{\xi(n-1)}-2}
\end{aligned}
$$

The following example shows that the above bound on the degree of $G$ is best possible.

Example 2.4. For each $n \geq 1$, there exists $\left(G_{n}, \Omega_{n}\right) \in \mathcal{W}_{n}$ such that $\left|\Omega_{n}\right|=\xi(n)$.

Proof. For $n=1$ we choose $G_{1}=\operatorname{Alt}(5)$ and the natural Alt(5)-set $\Omega_{1}$. Suppose that we have already found $\left(G_{n-1}, \Omega_{n-1}\right) \in \mathcal{W}_{n-1}$ with $\left|\Omega_{n-1}\right|=\xi(n-1)$. Then $G_{n}=\operatorname{Alt}(5) \operatorname{wr}_{\Omega_{n-1}} G_{n-1}$ acts primitively on $\Omega_{n}=\Omega_{1} \Omega_{n-1}$ in product action, and the degree of this representation is $\xi(n)$.

Consider some $(G, \Gamma) \in \mathfrak{W}_{n}$. In the sequel,

$$
1=K_{n} \leq K_{n-1} \leq \cdots \leq K_{1} \leq K_{0}=G
$$

shall denote the ascending socle series in $G$, i.e., $K_{\ell-1} / K_{\ell}=\operatorname{soc}\left(G / K_{\ell}\right)$ for $\ell=n, \ldots, 1$. Each $K_{\ell}$ is characteristic in $G$, and $K_{\ell-1} / K_{\ell}$ is the unique minimal normal subgroup in $G / K_{\ell}$.

For $k$-tuples $g=\left(g_{1}, \ldots, g_{k}\right)$ and $h=\left(h_{1}, \ldots, h_{k}\right)$ in $G^{k}$, we shall write $g \equiv$ $h\left(\bmod K_{\ell}\right)$ in order to express, that $g_{i} K_{\ell}=h_{i} K_{\ell}$ for $i=1, \ldots, k$.

We are now in a position to prove the main theorem of this section.

Theorem 2.5. Consider a reduced word $w$ of length $n>0$ in the free group $F_{k}$ of rank $k$. Let $(G, \Gamma) \in \mathcal{W}_{d+n}$ for some $d \in \mathbb{N}$. Then, for any $y=\left(y_{1}, \ldots, y_{k}\right)$ in $G^{k} \backslash K_{d}{ }^{k}$, there exist $g=\left(g_{1}, \ldots, g_{k}\right) \in G^{k}$ and $\gamma \in \Gamma$ such that

- $g \equiv y\left(\bmod K_{d}\right)$,

- the elements $\gamma_{j}=\gamma w_{j}(g)$ are pairwise distinct for $j=0, \ldots, n$.

In particular, $w(g) \neq 1$. 
Proof. If $k>n$, then at least $k-n$ variables do not appear in the reduced form of $w$. We may therefore assume that $k \leq n$. It is also easy to check that the result is true for $n \leq 2$. Let $n \geq 3$ and assume by induction, that the statement holds for words of length at most $n-1$. By Lemma 2.2 the group $G=S \mathrm{wr}_{\Delta} H$ acts either in diagonal action or in product action, with respect to a suitable primitive representation of $S$, or its socle is regular. Notice that $\operatorname{soc}(G)=K_{d+n-1}$ is the base subgroup $B$ of the wreath product. Another fact which will be used frequently in the sequel is that, if $h \equiv y\left(\bmod K_{d}\right)$ for some $h \in H^{k}$, then $b h \equiv y\left(\bmod K_{d}\right)$ for every $b \in B^{k}$.

Our induction provides elements $h=\left(h_{1}, h_{2}, \ldots, h_{k}\right) \in H^{k}$ and $\delta \in \Delta$ such that $h \equiv y\left(\bmod K_{d}\right)$ and such that the elements $\delta_{i}=\delta w_{i}(h)$ are pairwise distinct for $i=0, \ldots, n-1$. Choose any faithful transitive $S$-set $\Omega$ and let $G$ act on $\Omega \times \Delta$ in the natural way. The remark in the first paragraph of the proof of [2, Lemma 4] shows that we can find $\varepsilon=(\omega, \delta) \in \Omega \times \Delta$ and a subset $\varepsilon \subseteq B^{k}$ containing at least

$$
|S|^{|\Delta|(k-1)}|S|^{|\Delta|-1}(|S|-1)=|S|^{|\Delta| k}\left(1-\frac{1}{|S|}\right)=|B|^{k}\left(1-\frac{1}{|S|}\right)
$$

elements such that, whenever $b \in \mathcal{E}$, then $\varepsilon w_{i}(b h) \neq \varepsilon w_{j}(b h)$ for $0 \leq i<j \leq n$. In particular, $w_{i}(b h) \neq w_{j}(b h)$ for $0 \leq i<j \leq n$ and $b \in \mathcal{E}$.

Consider any primitive faithful $G$-set $\Gamma$. We aim to show that, for each $b \in \mathcal{E}$, we can find some $\gamma \in \Gamma$ such that the element $x_{i j}=w_{i}(b h) w_{j}(b h)^{-1}$ does not belong to $G_{\gamma}$ for $0 \leq i<j \leq n$. Assume by way of contradiction that this does not hold for a certain $b \in \mathcal{E}$. It follows that $\Gamma$ is the union of the sets

$$
\Gamma_{i j}=\left\{\gamma \mid \gamma x_{i j}=\gamma\right\} .
$$

The number of these sets is $\left(\begin{array}{c}n+1 \\ 2\end{array}\right)$. Hence there exists a pair $(i, j)$ such that

$$
\mu=\frac{|\Gamma|}{\left(\begin{array}{c}
n+1 \\
2
\end{array}\right)} \leq\left|\Gamma_{i j}\right| .
$$

Therefore $x_{i j}$ fixes at least $\mu$ elements from $\Gamma$. The desired contradiction will now be derived separately in each of the three cases furnished by Lemma 2.2.

Case 1: Product action. In this case, $G$ acts on $\Gamma=\Sigma^{\Delta}$, where $\Sigma$ is a primitive faithful $S$-set. We shall try to estimate the size of the set of fixed points fix $\Gamma(g)$ for a non-trivial element $g=f x \in G$ (with $f \in B, x \in H$ ). Recall that $g$ acts on $\Gamma$ via $(\varphi g)(\delta)=\varphi\left(\delta x^{-1}\right) f\left(\delta x^{-1}\right)$ for all $\varphi: \Delta \rightarrow \Sigma$.

Suppose first that $x^{-1}=\left(\delta_{1} \delta_{2} \ldots \delta_{\ell}\right)$ is a non-trivial cycle. If $\varphi: \Delta \rightarrow \Sigma$ is a fixed point for $g$, and if $\delta \in \operatorname{fix}_{\Delta}(x)$, then $\varphi(\delta) \in \operatorname{fix}_{\Sigma}(f(\delta))$. Moreover, we have $\varphi\left(\delta_{i+1}\right) f\left(\delta_{i+1}\right)=\varphi\left(\delta_{i}\right)$ for $i=1, \ldots, \ell-1$ and $\varphi\left(\delta_{\ell}\right) f\left(\delta_{\ell}\right)=\varphi\left(\delta_{1}\right)$. 
Therefore, if we want to construct an element $\varphi \in \operatorname{fix}_{\Gamma}(g)$, then we should, first of all, choose $\varphi(\delta) \in \operatorname{fix}_{\Sigma}(f(\delta))$ whenever $\delta \notin \operatorname{supp}_{\Delta}(x)$. Then, in order to define $\varphi$ on $\operatorname{supp}_{\Delta}(x)$, we can choose freely the value $\varphi\left(\delta_{1}\right)$ (this allows $|\Sigma|$ different choices), and then the value of $\varphi$ on the other points $\delta_{i}$ is determined by the above relations. However, not every such choice is admissible, since the $\ell$-th relation $\varphi\left(\delta_{\ell}\right) f\left(\delta_{\ell}\right)=\varphi\left(\delta_{1}\right)$ might not hold. We conclude that

$$
\left|\operatorname{fix}_{\Gamma}(g)\right| \leq|\Sigma| \cdot\left(\prod_{\delta \in \operatorname{fix}_{\Delta}(x)}\left|\operatorname{fix}_{\Sigma}(f(\delta))\right|\right) .
$$

It is now an easy matter to work out the general case: If $x$ has $r$ non-trivial cycles in its action on $\Delta$, then

$$
\left|\operatorname{fix}_{\Gamma}(g)\right| \leq|\Sigma|^{r} \cdot\left(\prod_{\delta \in \operatorname{fix}_{\Delta}(x)}\left|\operatorname{fix}_{\Sigma}(b(\delta))\right|\right) \leq|\Sigma|^{r+\left|\mathrm{fix}_{\Delta}(x)\right|} .
$$

Suppose that $x \neq 1$. Since $n \geq 3$, the group $H$ is not an alternating group. Therefore [8, Corollary 3$]$ yields $\operatorname{supp}_{\Delta}(x) \geq 2(\sqrt{|\Delta|}-1)$, whence

$$
\mid \text { fix }_{\Delta}(x)|\leq| \Delta \mid-2(\sqrt{|\Delta|}-1) .
$$

Now $r \leq \frac{|\Delta|-\left|\mathrm{fix}_{\Delta}(x)\right|}{2}$. Hence

$$
\begin{gathered}
r+\left|\operatorname{fix}_{\Delta}(x)\right| \leq \frac{|\Delta|+\left|\mathrm{fix}_{\Delta}(x)\right|}{2} \leq|\Delta|-\sqrt{|\Delta|}+1, \\
\left|\operatorname{fix}_{\Gamma}(g)\right| \leq|\Sigma|^{|\Delta|-\sqrt{|\Delta|}+1} .
\end{gathered}
$$

Suppose now that $x=1$ and $g=f \in B$. In this situation it is clear that

$$
\mid \text { fix }\left._{\Gamma}(g)|\leq(|\Sigma|-3)| \Sigma\right|^{|\Delta|-1},
$$

corresponding to the case when $S$ is an alternating group in natural action, $f$ has a single non-trivial entry and this entry is a 3-cycle.

As in the proof of [2, Lemma 3] at most one of the elements $x_{i j}$ (say $x_{0 n}$ ) has trivial top component, so that the set

$$
\Gamma_{0}=\bigcup_{\{i, j\} \neq\{0, n\}} \Gamma_{i j} \supseteq \Gamma \backslash \Gamma_{0 n}
$$

has size $\left|\Gamma_{0}\right| \geq|\Sigma|^{|\Delta|}-(|\Sigma|-3)|\Sigma|^{|\Delta|-1}=3|\Sigma|^{|\Delta|-1}$. An application of the pigeonhole principle yields that there exists a 2 -set $\{i, j\} \neq\{0, n\}$ for which $\Gamma_{i j}$ has size at least

$$
\mu_{0}=\frac{3|\Sigma|^{|\Delta|-1}}{\left(\begin{array}{c}
n+1 \\
2
\end{array}\right)-1}
$$

The inequality $\mu_{0} \leq|\Sigma|^{|\Delta|-\sqrt{|\Delta|}+1}$ now implies $|\Sigma|^{\sqrt{|\Delta|}-2} \leq\left(\begin{array}{c}n+1 \\ 2\end{array}\right)-1$. But this contradicts Lemma 2.3 . 
Case 2: Diagonal action. In this case, the set $\Gamma$ can be identified with the set of right cosets in $B=S^{\Delta}$ of the diagonal subgroup $D$ of $B$. As before, we try to describe $\operatorname{fix}_{\Gamma}(g)$ for $g=f x \in G$ (with $f \in B, x \in H$ ). Recall that the action of $G$ on $\Gamma$ is induced from the product action of $G$ on $B$ with respect to the right regular representation of $S$ on $\Sigma=S$.

If $g$ fixes $D t$ (where $t \in B$ ), then there exists $d \in S$ such that $(t f) x=\bar{d} t$, where $\bar{d}$ indicates the element of $D$, whose entries are all equal to $d$. Thus,

$$
t(\delta) f(\delta)=d t(\delta x)
$$

for every $\delta \in \Delta$. In particular, $f(\delta)=d^{t(\delta)}$ whenever $\delta \in$ fix $_{\Delta}(x)$.

We shall now use this information in order to count the elements in $\operatorname{fix}_{\Gamma}(g)$. There are at most $|S|$ possible choices for $d$. Once $d$ has been selected, each $t(\delta)$ $\left(\delta \in \operatorname{fix}_{\Delta}(x)\right)$ can be chosen in $\left|C_{S}(d)\right|$ different ways. Let $\chi(x)$ denote the set of $x$-orbits $c$ in $\Delta$ with length $\ell(c)>1$. If $c=\left(\delta_{1} \delta_{2} \ldots \delta_{\ell(c)}\right) \in \chi$, then, for any choice of $t\left(\delta_{1}\right)$, the values $t\left(\delta_{i}\right)(i=2, \ldots, \ell(c))$ are uniquely determined by the equation $t\left(\delta_{i}\right) f\left(\delta_{i}\right)=d t\left(\delta_{i+1}\right)$. Moreover, also the equation

$$
t\left(\delta_{\ell(c)}\right) f\left(\delta_{\ell(c)}\right)=d t\left(\delta_{1}\right)
$$

must hold, and it is not difficult to see, that this happens if and only if

$$
t\left(\delta_{1}\right)=d^{-\ell(c)} t\left(\delta_{1}\right) f\left(\delta_{1}\right) \ldots f\left(\delta_{m}\right) .
$$

Thus $t\left(\delta_{1}\right)$ must satisfy

$$
\left(d^{\ell(c)}\right)^{t\left(\delta_{1}\right)}=f\left(\delta_{1}\right) \ldots f\left(\delta_{\ell(c)}\right) .
$$

Hence the number of possible choices for $t\left(\delta_{1}\right)$ is $\left|C_{S}\left(d^{\ell(c)}\right)\right|$. Altogether we conclude that

$$
\left|\operatorname{fix}_{\Gamma}(g)\right| \leq\left.|S|\left|C_{S}(d)\right|^{\mid f i x} \Delta(x)\left|\prod_{c \in \chi(x)}\right| C_{S}\left(d^{\ell(c)}\right)|\leq| S\right|^{1+\left|\operatorname{fix}_{\Delta} x\right|+|\chi(x)|} .
$$

If $x \neq 1$, then the same arguments as in Case 1 (product action) yield

$$
\left|\operatorname{fix}_{\Gamma}(g)\right| \leq|S|^{|\Delta|-\sqrt{|\Delta|}+2} \text {. }
$$

On the other hand, if $x=1$ but $f \neq 1$, then we obtain that all values of $f$ must lie in the conjugacy class of a single non-trivial element $d \in S$, and that we have $\left|C_{S}(d)\right|$ choices for each of the values $t(\delta)(\delta \in \Delta)$. Moreover, if $t_{1}$ and $t_{2}$ have been obtained in this way, then $t_{1} t_{2}^{-1} \in C_{S}(d)^{\Delta}$. In particular, $D t_{1}=D t_{2}$ if and only if $t_{1} t_{2}^{-1} \in D \cap C_{S}(d)^{\Delta}$. Therefore,

$$
\left|\operatorname{fix}_{\Gamma}(g)\right| \leq\left|C_{S}(d)\right|^{|\Delta|-1} \leq|S|^{|\Delta|-1} .
$$

Considering $\Gamma_{0}$ and $\mu_{0}$ as in Case 1 (product action), we find that

$$
\left|\Gamma_{0}\right| \geq|S|^{|\Delta|}-|S|^{|\Delta|-1}=|S|^{|\Delta|-1}(|S|-1)
$$


and

$$
\mu_{0}=\frac{|S|^{|\Delta|-1}(|S|-1)}{\left(\begin{array}{c}
n+1 \\
2
\end{array}\right)-1} \leq|S|^{|\Delta|-\sqrt{|\Delta|}+2} .
$$

This leads to the contradiction $|S|^{\sqrt{|\Delta|}-3}(|S|-1) \leq\left(\begin{array}{c}n+1 \\ 2\end{array}\right)-1$ (cf. Lemma 2.3).

Case 3: The socle acts regularly. Now we have $\Gamma=B$ and $G=B X$, where $B=S^{\Delta}$ acts regularly on $\Gamma$ and $X$ is a point stabilizer. In order to avoid confusion, the action of $s \in G$ on $\gamma \in \Gamma$ shall be denoted by $\gamma . s$. As before, we consider $g=f x \in G$ with $f \in B, x \in X$ and try to count the number of its fixed points. Notice that $x$ might not belong to $H$. Therefore $x=b h$ with $b \in B, h \in H$.

Now $\gamma \in \operatorname{fix}_{\Gamma}(g)$ if and only if $\gamma=\gamma \cdot g=\gamma \cdot(f b h)$ if and only if $\gamma \cdot h^{-1}=\gamma f b$. The latter means that

$$
\gamma(\delta h)=\gamma(\delta) f(\delta) b(\delta) \quad \text { for all } \delta \in \Delta .
$$

If $h$ fixes $\delta$, then $f(\delta) b(\delta)=1$ and $\gamma(\delta)$ can take any value in $S$. If $\left(\delta_{1} \delta_{2} \ldots \delta_{m}\right)$ is an $h$-orbit, then $\gamma\left(\delta_{i+1}\right)$ is determined by $\gamma\left(\delta_{i}\right)$ via

$$
\gamma\left(\delta_{i+1}\right)=\gamma\left(\delta_{i}\right) f\left(\delta_{i}\right) b\left(\delta_{i}\right) \text { for } i=1, \ldots, m-1,
$$

but we can choose $\gamma\left(\delta_{1}\right)$. With $r$ denoting the number of non-trivial $h$-orbits in $\Delta$, we obtain

$$
\left|\operatorname{fix}_{\Gamma}(g)\right| \leq|S|^{\mid \text {fix }_{\Delta} h \mid+r} \leq|S|^{|\Delta|-\sqrt{|\Delta|}+1}
$$

as in Case 1 (product action) for elements $g$ satisfying $h \neq 1$. However, any nontrivial $g \in G$ either has no fixed-point in $\Gamma$ or satisfies $h \neq 1$, because $B$ acts regularly on $\Gamma$. In particular,

$$
\mu=\frac{|S|^{|\Delta|}}{\left(\begin{array}{c}
n+1 \\
2
\end{array}\right)} \leq\left|\Gamma_{i j}\right| \leq|S|^{|\Delta|-\sqrt{|\Delta|}+1} \quad \text { and } \quad|S|^{\sqrt{|\Delta|}-1} \leq\left(\begin{array}{c}
n+1 \\
2
\end{array}\right)
$$

a contradiction to Lemma 2.3.

A closer look at the proof of the above result, gives information on the number of $k$-tuples $g$ which satisfy the assertion of Theorem 2.5 .

Corollary 2.6. In the notation of Theorem 2.5 , let

$$
G=S_{n} \operatorname{wr}_{\Delta_{n}}\left(S_{n-1} \mathrm{wr}_{\Delta_{n-1}}\left(\ldots\left(S_{1} \mathrm{wr}_{\Delta_{1}} H\right) \ldots\right)\right)
$$

where $\left(H, \Delta_{1}\right) \in \mathcal{W}_{d}$ and $S_{1}, \ldots, S_{n}$ are non-abelian finite simple groups. If $\left|S_{i}\right|=s_{i}$ for all $i$ and $|G|=r|H|$, then the number of $k$-tuples $g \in G^{k}$ satisfying the statement of Theorem 2.5 is at least

$$
r^{k} \cdot \prod_{i=1}^{n}\left(1-\frac{1}{s_{i}}\right)
$$


Proof. Clearly, $G=K_{d} H$. Suppose first that $w(y) \neq 1$. Then, for every $b \in K_{d}{ }^{k}$, the $k$-tuple $g=b y$ satisfies $w(g) \neq 1$ too. The number of such tuples is $r^{k}$. Suppose next, that $w(y)=1$. Following the recursive procedure in the proof of Theorem 2.5, the required elements are constructed in $n$ steps. In the $i$-the step, every $k$-tuple constructed so far, can be extended in at least $s_{i}{ }^{\left|\Delta_{i}\right|}\left(1-\frac{1}{s_{i}}\right)$ different ways. Since $\prod_{i=1}^{n} s_{i}{ }^{\left|\Delta_{i}\right|}=\left|K_{d}\right|$, the claim follows in this case too.

\section{Ubiquity of free subgroups in certain profinite groups}

Let $\delta=\left\{S_{i} \mid i \geq 1\right\}$ be a family of non-abelian finite simple groups. We define recursively a sequence of groups and permutation representations as follows:

- $W_{1}=S_{1}$ and $\Delta_{1}$ is any faithful primitive $W_{1}$-set,

- $W_{n}=S_{n} \operatorname{wr}_{\Delta_{n-1}} W_{n-1}$ and $\Delta_{n}$ is any faithful primitive $W_{n}$-set for all $n \geq 1$.

Clearly, $\left(W_{n}, \Delta_{n}\right) \in W_{n}$ for all $n$, and there are the natural surjective homomorphisms $\rho_{n}: W_{n} \rightarrow W_{n-1}$. We can thus form the inverse limit $W=\lim \left(W_{n}, \rho_{n}\right)$, which is a profinite group. A basis of open neighborhoods of the identity in $W$ is given by the subgroups $K_{n}=\operatorname{ker}\left(\pi_{n}\right)$, where $\pi_{n}: W \rightarrow W_{n}$ denotes the natural projection. We shall denote the class of groups constructed in this way by $\mathcal{W}(\delta)$.

For every $w \in F_{k}$, let

$$
C(w)=\left\{h \in W^{k} \mid w(h) \neq 1\right\} \quad \text { and } \quad C=\bigcap_{1 \neq w \in F_{k}} C(w) .
$$

If $h=\left(h_{1}, \ldots, h_{k}\right) \in C$, then $\left\langle h_{1}, \ldots, h_{k}\right\rangle$ is free of rank $k$, because $w(h) \neq 1$ for every non-trivial $w \in F_{k}$. Thus $C=W(k)$. It is our aim to prove that $W(k)$ is a rather large subset of $W^{k}$. Recall that a meagre subset of a topological space is a countable union of nowhere dense subsets (i.e., of subsets, whose closure has empty interior).

Theorem 3.1. If $\&$ is a set of non-abelian finite simple groups, and if $W \in \mathcal{W}(8)$, then the set $W(k)$ is dense in $W^{k}$ and its complement is a meagre subset of $W^{k}$.

Proof. Consider a non-trivial $w \in F_{k}$. The evaluation map $\mu_{w}: W^{k} \rightarrow W$, which sends every $h \in W^{k}$ to $w(h) \in W$, is continuous. Moreover, $W$ is a Hausdorff space. Therefore $\mu_{w}{ }^{-1}(1)$ is closed and $C(w)=W^{k} \backslash \mu_{w}{ }^{-1}(1)$ is open.

Let $n$ be the length of $w$ and choose a non-trivial element $y \in W^{k}$. There exists $d$ such that $y \notin K_{d}{ }^{k}$. An application of Theorem 2.5 gives $h \in W^{k}$ such that $h \equiv y\left(\bmod K_{d}\right)$ and $w(h) \notin K_{d+n}$. In particular, $w(h) \neq 1$, so that $h \in C(w)$. On the other hand $h K_{d}=y K_{d}$, so that the intersection of the open neighborhood $y K_{d}$ of $y$ with $C(w)$ is non-empty. This shows that $C(w)$ is dense in $W^{k}$. 
Since $W^{k}$ is a compact Hausdorff space, Baire's theorem yields that $W(k)$ is dense, being a countable intersection of open dense subsets. Finally,

$$
W^{k} \backslash W(k)=W^{k} \backslash \bigcap_{1 \neq w \in F_{k}} C(w)=\bigcup_{1 \neq w \in F_{k}}\left(W^{k} \backslash C(w)\right) .
$$

Since each $C(w)$ is open and dense, $W^{k} \backslash C(w)$ is nowhere dense. This proves the claim.

Theorem 3.1 has the following consequence.

Corollary 3.2. Suppose that 8 is a set of non-abelian finite simple groups. Then every $W \in \mathcal{W}(8)$ contains $2^{\boldsymbol{N}_{0}}$ free subgroups of rank $2^{\boldsymbol{\aleph}_{0}}$.

Proof. The group $W$ is metrizable and complete. Therefore it is a Polish space. Since $W$ is not discrete, [6, Theorem 1] yields that $W$ contains a dense free subgroup. The claim now follows from [6, Corollary 3].

The largeness of $W(k)$ can also be considered from a measure-theoretic point of view, because every group in $W(\delta)$ is a measure space with respect to its canonical Haar measure. Since each compact group has finite volume, we may choose the normalized Haar measure and consider the group as a probability space.

The arguments contained in [2, Section 5] can be applied, without any change, in our set up. This gives the following result.

Theorem 3.3. Let 8 be a set of non-abelian finite simple groups, and let $W \in 8$. Then the set $W^{k} \backslash W(k)$ has measure zero with respect to the normalized Haar measure of $W$.

We finish this article with two open problems.

Questions 3.4. Suppose that the profinite group $W$ is formed from iterated wreath products as above, but without further restrictions to the nature of the faithful $W_{n}$-sets (i.e., we do not necessarily require that these sets are primitive).

- Is it still true then that $W^{k} \backslash W(k)$ is meagre?

- Is it still true then that $W^{k} \backslash W(k)$ has measure zero with respect to the normalized Haar measure?

In order to answer these questions, we would need a stronger form of Theorem 2.5. Namely it would be useful to know that the claim of Theorem 2.5 holds when the wreath products considered are defined without restrictions on the faithful permutation representations used at each stage. Although we have been unable 
to supply a proof of this stronger result, we believe that the answers to the above questions are positive.

Acknowledgments. The second author wishes to thank the University of Mainz for financial support and for the warm hospitality during the period in which the research reported in this paper was carried out.

\section{Bibliography}

[1] M. Abért, Group laws and free subgroups of topological groups, Bull. Lond. Math. Soc. 37 (2005), no. 4, 525-534.

[2] M. Bhattacharjee, The ubiquity of free subgroups in certain inverse limits of groups, J. Algebra 172 (1995), 134-146.

[3] J. D. Dixon, Most finitely generated permutation groups are free, Bull. Lond. Math. Soc. 22 (1990), 222-226.

[4] J.D. Dixon and B. Mortimer, Permutation Groups, Grad. Texts in Math. 163, Springer, New York, 1996.

[5] D. B. A. Epstein, Almost all subgroups of a Lie group are free, J. Algebra 19 (1971), 261-262.

[6] P. M. Gartside and R. W. Knight, Ubiquity of free subgroups, Bull. Lond. Math. Soc. 35 (2003), 624-634.

[7] J. Lafuente, On restricted twisted wreath products of groups, Arch. Math. 43 (1984), 208-209.

[8] M.W. Liebeck and J. Saxl, Minimal degrees of primitive permutation groups, with an application to monodromy groups of covers of Riemann surfaces, Proc. Lond. Math. Soc. (3) 63 (1991), 266-314.

[9] H.D. Macpherson, Groups of automorphisms of $\aleph_{0}$-categorical structures, $Q . J$. Math. 37 (1986), 449-465.

[10] M. Quick, Probabilistic generation of wreath products of non-abelian finite simple groups, Comm. Algebra 32 (2004), 4753-4768.

[11] M. Quick, Probabilistic generation of wreath products of non-abelian finite simple groups. II, Int. J. Algebra Comput. 16 (2006), 493-503.

[12] C. D. Reid, Inverse system characterizations of the (hereditarily) just infinite property in profinite groups, Bull. Lond. Math. Soc. 44 (2012), no. 3, 413-425.

[13] M. Vannacci, Finite generation of iterated wreath products in product action, Arch. Math. (Basel) 105 (2015), 205-214.

[14] M. Vannacci, On hereditarily just infinite profinite groups obtained via iterated wreath products, J. Group Theory 19 (2016), no. 2, 233-238. 
[15] J. S. Wilson, On just infinite abstract and profinite groups, in: New Horizons in Pro- $p$ Groups, Progr. Math. 184, Birkhäuser, Boston (2000), 219-246.

[16] J. S. Wilson, Large hereditarily just infinite groups, J. Algebra 324 (2010), 248-255.

Received April 18, 2016; revised December 30, 2016.

\section{Author information}

Felix Leinen, Institute of Mathematics, Johannes Gutenberg-University, 55099 Mainz, Germany.

E-mail: leinen@uni-mainz.de

Orazio Puglisi, Dipartimento di Matematica e Informatica "U. Dini”, Università di Firenze, Viale Morgagni 67A, 50134 Firenze, Italy.

E-mail: puglisi@math.unifi.it 